\title{
Akteure in Reallaboren - Reallabore als Akteure
}

\author{
Andreas Seebacher, Sophia Alcántara \& Alexandra Quint
}

In der praktischen Reallaborarbeit sind Überlegungen zu möglichen und Analysen von tatsächlichen Akteurskonstellationen hilfreich, um sich das Netzwerk zu vergegenwärtigen (s. auch Eckart et al. 2018). Mit den Akteuren und der Frage, wie sie miteinander verknüpft sind, steht und fällt der Erfolg eines Reallabors. Ihre Zahl, ihre Ziele, ihr Engagement, ihre Verfügbarkeit und das (sich im Laufe der Zeit wandelnde) Beziehungsgeflecht unter ihnen bestimmen, so die Erfahrung aus BaWü-Labs beider Förderlinien ${ }^{1}$, den Fortgang der Reallaborarbeit. Sich systematisch mit diesen Aspekten zu befassen, ist nicht nur in der Konzeptionsphase oder anlässlich wichtiger Meilensteine im Projektverlauf von Bedeutung, sondern empfiehlt sich auch zwischendurch, da dies trotz seiner Wichtigkeit im Trubel des Alltagsgeschäftes leicht aus dem Blickfeld gerät.

Zur Illustration der Akteurskonstellation in einem Reallabor wird hier das Bild dreier konzentrischer Kreise skizziert - ein idealisiertes Modell, basierend auf den Erfahrungen der Autorinnen und des Autors. Es ist als ein Vorschlag zu verstehen und soll als Anregung dienen, die stets reallabor-spezifisch unterschiedliche Systematik der Akteure aufzubereiten. Das Modell fasst die mögliche Akteurskonstellation innerhalb eines Reallabors wie folgt: Um den Kernbereich (als innerstem Kreis) legt sich ringförmig ein innerer (Akteurs-)Kreis, der wiederum von einem äußeren (Akteurs-)Kreis umschlossen wird (s. Abb. 1).

Wer aber ist in diesem Zusammenhang mit ,Akteur' gemeint? ${ }^{2}$ Sehr allgemein beschrieben sind damit individuell oder kollektiv sozial Handelnde (Gabriel 2004) gemeint, die als natürliche oder juristische Personen, als nicht-organisierte Gruppen oder als öffentlich-rechtlich verfasste Akteure auftreten. Mit Blick auf die außerwissenschaftlichen Akteure, die als Praxisakteure und - mehr noch - als Praxispartner für die Reallaborarbeit von besonderer Bedeutung sind, lassen sich

1 In Baden-Württemberg wurden ab 2015 in den zwei Förderlinien „Reallabore“ und „Reallabore Stadt“ insgesamt 14 Reallabore (die „BaWü-Labs“) gefördert.

$2 \mathrm{Zu}$ den Herausforderungen der Begrifflichkeit s. a. Defila und Di Giulio (2018).

(C) Der/die Herausgeber bzw. der/die Autor(en) 2018

R. Defila und A. Di Giulio (Hrsg.), Transdisziplinär und

transformativ forschen, https://doi.org/10.1007/978-3-658-21530-9_9 


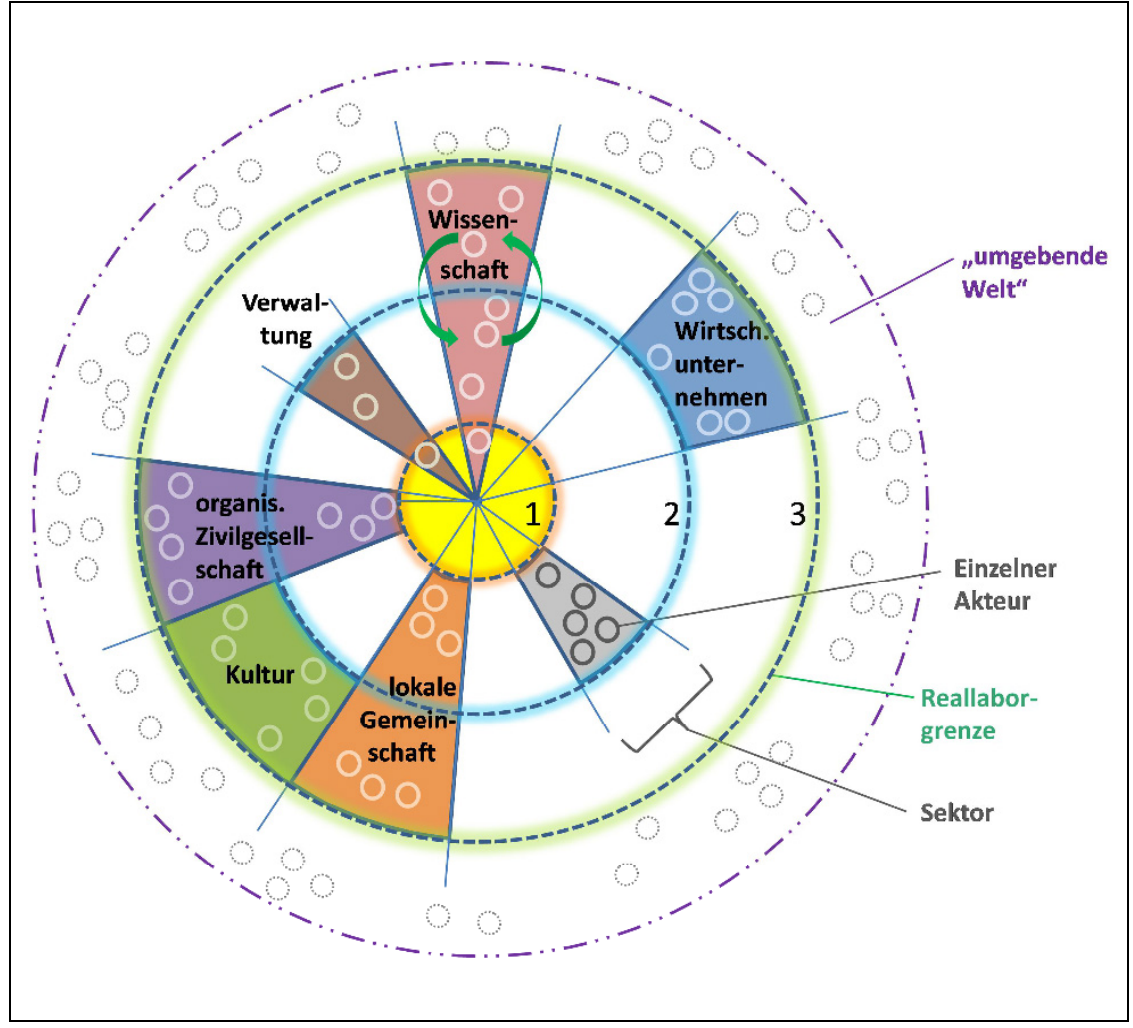

Abbildung 1: Das 3-Kreise-Modell der Akteurskonstellation in einem Reallabor. Den Kernbereich (1) nehmen die für Konzeption, Kohärenz, Prozessgestaltung und Steuerung verantwortlichen Akteure ein. Zum inneren (Akteurs-)Kreis (2) gehören die Akteure, die besonders intensiv an den Reallaboraktivitäten mitwirken. Im äußeren (Akteurs-)Kreis (3) befinden sich die Akteure, die sich punktuell an Reallaboraktivitäten beteiligen. Akteure, die demselben Kontext angehören (Akteurstypen), bilden Sektoren, die im Regelfall kreisübergreifend sind und sich im Idealfall über alle drei Kreise erstrecken. Die Kreise haben durchlässige Grenzen, auch nach außen (zur umgebenden Welt), und erlauben Fluktuation (grüne Pfeile); möglicherweise erlauben auch die Grenzen zwischen Sektoren Fluktuation. Die schematische Darstellung zeigt beispielhaft eine Momentaufnahme einer möglichen Akteurskonstellation. 
in der Gesellschaft Akteurstypen ausmachen, die sehr unterschiedlichen Kontexten zugerechnet werden können. Solche Kontexte können zum Beispiel sein: „organisierte Zivilgesellschaft, lokale Gemeinschaft, (...) Verwaltung, Wirtschaftsunternehmen, Politik, Kultur, Bildung" (vgl. Arnold und Piontek 2018, S. 148). Diese Typisierung kann jedoch nur als grobe Folie gedacht werden, vor deren Hintergrund für das einzelne Reallabor eine Feineinteilung vorgenommen werden kann und sollte. Jeder Typus von Akteur (beispielsweise „Wirtschaftsunternehmen") ist zu verstehen als eine Menge aus einzelnen Akteuren (hier z. B.: einzelne Firmen), die einem übergeordneten Merkmal entsprechen. Im (Reallabor-)Projektalltag entsteht die Vielfalt der Akteure nun gerade durch die vielen einzelnen Akteure, die sich hinsichtlich einer Fülle von Kriterien fein voneinander unterscheiden.

Die einzelnen wissenschaftlichen und außerwissenschaftlichen Akteure lassen sich zu jedem Beobachtungszeitpunkt je nach Grad ihres Mitentscheidens und ihrer Mitwirkung (beispielsweise hinsichtlich des Personalaufwandes, der Intensität und Dauer der Mitwirkung oder der Menge an Detailwissen) den drei Kreisen zuordnen. Die Grenzen zwischen den Kreisen sind nach diesem Modell durchlässig und erlauben Fluktuation. Dies gilt auch für die ,Außengrenze', an der sich das Reallabor und die umgebende Welt berühren. Den Kernbereich macht ein für Konzeption, inhaltliche Kohärenz, Prozessgestaltung und organisatorische Steuerung verantwortliches Team aus, dem idealerweise sowohl wissenschaftliche Akteure aus verschiedenen Disziplinen als auch außerwissenschaftliche Akteure, hier auch als „Praxisakteure“ bezeichnet, aus unterschiedlichen Kontexten angehören. Im Kernbereich vieler BaWü-Labs sind tatsächlich vor allem Wissenschaftler(innen) anzutreffen. Der Kernbereich lässt sich als der Ort begreifen, an dem am intensivsten geplant, an der Konzipierung von Experimenten gearbeitet und geforscht wird. Der um dieses Zentrum herum angeordnete innere (Akteurs-)Kreis ist der Ort, an dem vor allem von und mit den Praxispartnern besonders intensiv experimentiert wird. Er beinhaltet all jene Akteure, die über einen längeren Zeitraum hinweg kontinuierlich, intensiv und unter größerem Ressourceneinsatz (Zeit, Geld, Material) an den Reallaboraktivitäten mitwirken. Die Praxisakteure im inneren Kreis (wie auch im Kernbereich) werden bei einigen BaWü-Labs auch als Praxispartner bezeichnet, um die aus der engen Kooperation herrührende Sonderstellung zu betonen. Der den inneren Kreis umschließende äußere (Akteurs-)Kreis schließlich wird gebildet von all jenen Akteuren, die sich eher diskontinuierlich, eher anlassbezogen, eher über einen kürzeren Zeitraum und/oder eher mit geringerem Ressourceneinsatz in die Reallaborarbeit einbringen; hierzu ließe sich auch die „Cloud“ (Parodi et al. 2016, S. 14) der digitalen Fangemeinde rechnen. Dieser Darstellung folgend werden interne Akteure (Kernbereich und innerer Kreis) und externe Akteure (äußerer Kreis) unterschieden. 
Idealerweise würde man sich im Sinne von Co-Design und Co-Produktion wünschen, dass sich in allen drei genannten Kreisen jeweils Akteure aus allen für ein Reallabor relevanten Kontexten befinden. Das wären dann im hier skizzierten Modell tortenstückförmige Sektoren, die im Regelfall mehrere Kreise erfassen. In den Sektoren sollten vor allem wichtige Schlüsselakteure vertreten sein: sowohl Akteure, die dem Anliegen des jeweiligen Reallabors thematisch besonders nahestehen (z. B. bestimmte Mobilitätsakteure beim Reallabor für nachhaltige Mobilitätskultur (BaWü-Lab RNM) oder einzelne Textilfirmen beim Reallabor Nachhaltige Transformation der Textilwirtschaft am Standort Dietenheim (BaWü-Lab Dietenheim zieht an)), als auch Akteure, die die für die Reallaborarbeit wichtigen politischen, organisatorischen und rechtlichen Bedingungen beeinflussen können. Zur Verfeinerung des Modells bzw. zur Klärung der Frage, in welchem der Kreise sich die Akteure die überwiegende Zeit hindurch befinden und ob (eventuell auch: warum) sie zwischen ihnen fluktuieren, können systematische Netzwerkanalysen beitragen, die die Akteurskonstellation zu Beginn eines Reallabors, während seines Verlaufes und gegen Ende beschreiben.

Mit diesem Netzwerk von (teils vertraglich) miteinander verbundenen ReallaborAkteuren entsteht ein Gebilde, das als Gesamtheit der Vorstellung eines quasiinstitutionellen Ensembles nahekommt, das nach außen hin wie ein Akteur auftritt. Ein Reallabor fordert und fördert nicht nur Partizipation bei der Gestaltung transformativer Prozesse (Parodi et al. 2016, S. 16; Wagner und Grunwald 2015; Schneidewind 2015), sondern partizipiert auch selbst aktiv an diesen Prozessen. Auf der Bühne der ,realen Welt' kommt einem Reallabor demnach ebenfalls die Rolle eines Akteurs zu, der auf sein Projektgebiet einwirkt. Ein Reallabor, als Summe seiner vielen Akteure und als eine abgegrenzte Einheit begriffen, kann nach außen hin als ein Akteur auftreten - ein Akteur unter vielen, der legitimerweise das gleiche Recht in Anspruch nimmt (vgl. dazu aber auch Eckart et al. 2018), Wirklichkeit und Alltag zu beeinflussen wie andere gesellschaftliche Akteure neben ihm.

\section{Literatur}

Arnold, A., \& Piontek, F. M. (2018). Zentrale Begriffe im Kontext der Reallaborforschung. In R. Defila \& A. Di Giulio (Hrsg.), Transdisziplinär und transformativ forschen. Eine Methodensammlung (S. 143-154). Wiesbaden: Springer VS.

Defila, R., \& Di Giulio, A. (2018). Reallabore als Quelle für die Methodik transdisziplinären und transformativen Forschens - eine Einführung. In R. Defila \& A. Di Giulio (Hrsg.), Transdisziplinär und transformativ forschen. Eine Methodensammlung (S. 935). Wiesbaden: Springer VS. 
Eckart, J., Ley, A., Häußler, E., \& Erl, Th. (2018). Leitfragen für die Gestaltung von Partizipationsprozessen in Reallaboren. In R. Defila \& A. Di Giulio (Hrsg.), Transdisziplinär und transformativ forschen. Eine Methodensammlung (S. 105-135). Wiesbaden: Springer VS.

Gabriel, M. (Hrsg.) (2004). Paradigmen der akteurszentrierten Soziologie. Wiesbaden: Springer VS.

Parodi, O., Beecroft, R., Albiez, M., Quint, A., Seebacher, A., Tamm, K., \& Waitz, C. (2016). Von „Aktionsforschung“ bis „Zielkonflikte“. Schlüsselbegriffe der Reallaborforschung. Technikfolgenabschätzung - Theorie und Praxis, 25 (3), (S. 9-18).

Schneidewind, U., \& Singer-Brodowski, M. (2015). Vom experimentellen Lernen zum transformativen Experimentieren - Reallabore als Katalysator für eine lernende Gesellschaft auf dem Weg zu einer Nachhaltigen Entwicklung. Zeitschrift für Wirtschaftsund Unternehmensethik (ZFWU), 16 (1), (S. 10-28).

Wagner, F., \& Grunwald, A. (2015). Reallabore als Forschungs- und Transformationsinstrument: Die Quadratur des hermeneutischen Zirkels. GAIA, 24 (1), (S. 26-31).

Open Access Dieses Kapitel wird unter der Creative Commons Namensnennung 4.0 International Lizenz (http://creativecommons.org/licenses/by/4.0/deed.de) veröffentlicht, welche die Nutzung, Vervielfältigung, Bearbeitung, Verbreitung und Wiedergabe in jeglichem Medium und Format erlaubt, sofern Sie den/die ursprünglichen Autor(en) und die Quelle ordnungsgemäß nennen, einen Link zur Creative Commons Lizenz beifügen und angeben, ob Änderungen vorgenommen wurden.

Die in diesem Kapitel enthaltenen Bilder und sonstiges Drittmaterial unterliegen ebenfalls der genannten Creative Commons Lizenz, sofern sich aus der Abbildungslegende nichts anderes ergibt. Sofern das betreffende Material nicht unter der genannten Creative Commons Lizenz steht und die betreffende Handlung nicht nach gesetzlichen Vorschriften erlaubt ist, ist für die oben aufgeführten Weiterverwendungen des Materials die Einwilligung des jeweiligen Rechteinhabers einzuholen. 